\title{
Fault-Tolerant Lyapunov-Gain-Scheduled PID Control of a Quadrotor UAV
}

\author{
Abderrahmen Bouguerra ${ }^{1}$, Djamel Saigaa ${ }^{2}$, Kamel Kara $^{3}$, Samir Zeghlache ${ }^{1}$ \\ ${ }^{1}$ Department of Electrical Engineering, M'sila University, Zip postal 166 Ichbilia 28000, M'sila, Algeria \\ ${ }^{2}$ Departments of Electronics, M'sila University, Zip postal 166 Ichbilia 28000, M'sila, Algeria \\ ${ }^{3}$ Departments of Electronics, Blida University, Zip postal 270 Blida, Algeria \\ * Corresponding author's Email:rah_bou@yahoo.fr
}

\begin{abstract}
The work has done in this paper concern the passive fault tolerant control. Based on Gain-Adaptive Proportional-Integral-Derivative (PID) using the approach from the theory of Lyapunov and their application to the model vertical flying drone Quadrotor type, the PID controller with fixed parameters may fail to provide acceptable control performance. To improve the PID control effect, new designs of the Lyapunov gain Scheduled PID controller (LGSPID) were presented in this paper. The proposed techniques were applied to the Quadrotor, where adaptive PID controllers were proposed for fault-tolerant control system in the presence of actuator faults. The parameters of PID controller were adjusted by an adaptation algorithm gradient type, used to tune in real-time the controller gain, the proposed adaptive PID controller was compared with the conventional PID. The obtained results confirm the effectiveness of the proposed method.
\end{abstract}

Keywords: Adaptation algorithm gradient type; Gain-adaptive PID; LGSPID; Quadrotor model; PID control.

\section{Introduction}

Fault-Tolerant Control (FTC) is a relatively new idea that makes possible to develop a control feedback that allows keeping the required system performance in the case of faults[1]. The control strategy can perceive fault tolerant when there is an adapta- tion mechanism that changes the control law in the case of faults.

Another solution is to use hardware redundancy in sensors and/or actuators. In general, FTC systems are classified into two distinct classes [2]: passive and active. In passive FTC [3, 4], controllers are designed to be robust against a set of presumed faults, therefore there is no need for fault detection. In contrast to passive ones, active FTC schemes react to system components faults actively by re- configuring control actions and by doing so the system stability and acceptable performance is maintained.

A Quadrotor is an aircraft that is lifted and propelled by four rotors. The Control of Quadrotor can be achieved by varying the relative speed of each a rotor to change the thrust and torque produced by each. Quadrotors are classified as rotorcraft, as opposed to fixed wing aircraft, because their lift is derived from four rotors [5].

PID controllers are the most familiar controller in the society of automation and control, due to their simple structure and wide variety of usage. These kinds of controllers are classified into two main categories in terms of parameters selection strategies. In the first group, controller gains are fixed during operation while in the second group, gains change based on the operating conditions.

In the first group, gains are tuned by the designer and remain invariable during the operation.

One of the most familiar methods for choosing control gains in this group is Ziegler-Nichols method [6].

In most applications, due to structural changes the controlled system may lose its effectiveness, therefore the PID gains need to be continuously retuned during the system life span. To reduce the effort of retuning the gains and also in order to 
increase system's performance, in the second group of controllers, the gains are adapted online.

A number of methods have been proposed in documents for PID gain scheduling [7]. A stable gain-scheduling PID controller is developed based on grid point concept for nonlinear systems. Different gain scheduling methods have been studied and compared $[8,9]$ and a new PID scheme is proposed in which the controller gains are scheduled by a fuzzy inference scheme. Many methods and research work in this domain in [10-14]. And an intelligent control scheme uses a fuzzy switching mechanism, grey prediction and genetic algorithm (GA) in [15]. The interested readers can find a brief review of different fuzzy PID structures in [16].

In this work, adaptive Lyapunov Gain Scheduled PID control approaches for a Quadrotor system was proposed.

A PID controller is used to approach a law unknown ideal control online. Contrary to the work of [17-19], wherein the adaptation law is selected to ensure the decrease of a Lyapunov function candidate on the output error, the adaptive law, in this work was selected to minimize the gradient method a quadratic criterion of error at the input of the system, the error between the unknown ideal control and output of the PID controller [17-19].

The remainder of this paper is organized as follows. The model of the Quadrotor is described in Section II. The Lyapunov Gain Scheduled PID (LGSPID) strategy is designed in Section III. Section IV presents the simulation results to demonstrate the effectiveness of the FTC Controller. Concluding remarks are provided in Section VI.

\section{Model of the auadrotor UAV}

In this section, the general dynamic model of a Quadrotor UAV was studied.

A body-fixed frame $B\left(O^{\prime}, x, y, z\right)$ and the earthfixed frame $E(O, X, Y, Z)$ were assumed to be at the center of gravity of the Quadrotor UAV, where the z-axis was pointing upwards, as seen in Figure 1.

The orientation of Quadrotor UAV that referred to as roll, pitch and yaw was given by a vector $(\varphi, \theta, \psi)$ which was measured with respect to the earth coordinate frame $E$.

Based on some basic assumptions as given below:

- Design is symmetrical.

- Quadrotor body is rigid.

- Propellers are rigid.

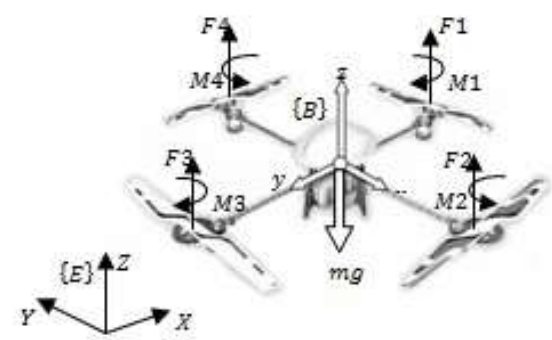

Figure1 The Quadrotor in an inertial frame

- Free stream air velocity is zero.

- The motors dynamics is relatively fast and can be neglected.

- The flexibility of the blade is relatively small and can be neglected.

- Drag is supposed to be linear, thus obeying Stokes's law.

The dynamic model [23] is:

$$
\left\{\begin{array}{l}
\ddot{x}=-\frac{u_{1}}{m} \sin \theta \\
\ddot{y}=\frac{u_{1}}{m} \cos \theta \cdot \sin \varphi \\
\ddot{z}=\frac{u_{1}}{m} \cos \theta \cdot \cos \varphi-g \\
\ddot{\varphi}=\tilde{\tau} \varphi \\
\ddot{\theta}=\tilde{\tau} \theta \\
\ddot{\psi}=\tilde{\tau} \psi
\end{array}\right.
$$

The generalized moments are:

$$
\begin{aligned}
& \tilde{\tau}=\left(\begin{array}{c}
\tilde{\tau}_{\psi} \\
\tilde{\tau} \theta \\
\tilde{\tau} \varphi
\end{array}\right) \\
& \ddot{\eta}=\tilde{\tau}
\end{aligned}
$$

where $\zeta=(x, y, z) \in \mathrm{R}^{3} \eta=(\varphi, \theta, \psi) \in \mathrm{S}^{3}$ and $\mathrm{J}=T_{\eta}^{T} \mathrm{I} T_{\eta}$.

$$
\begin{aligned}
& T_{\eta}=\left[\begin{array}{ccc}
-s \theta & 0 & 1 \\
c \theta s \phi & c \phi & 0 \\
c \theta c \phi & -s \phi & 0
\end{array}\right] \\
& \mathrm{I}=\left[\begin{array}{ccc}
I_{x x} & 0 & 0 \\
0 & I_{y y} & 0 \\
0 & 0 & I_{z z}
\end{array}\right]=\left[\begin{array}{ccc}
I_{x x} & 0 & 0 \\
0 & I_{x x} & 0 \\
0 & 0 & 2 I_{x x}
\end{array}\right]
\end{aligned}
$$


The coriolis and centripetal vector denoted by $C(\eta, \dot{\eta})$ defined as below and computed as given by (9).

$$
\begin{aligned}
& C(\eta, \dot{\eta})=\left(\dot{\mathrm{J}}-\frac{1}{2} \frac{\partial}{\partial \eta}\left(\dot{\eta}^{T} \mathrm{~J}\right)\right) \\
& \mathrm{J}=I_{x x}\left[\begin{array}{ccc}
1+c_{\theta}^{2} c_{\phi}^{2} & -c \theta s \phi c \phi & -s \theta \\
-c \theta s \phi c \phi & 2-c_{\phi}^{2} & 0 \\
-s \theta & 0 & 1
\end{array}\right] \\
& \dot{\mathrm{J}}=I_{x x}\left[\begin{array}{ccc}
\dot{\theta} s 2 \theta c_{\phi}^{2}+\dot{\phi} s 2 \phi c_{\theta}^{2} & \dot{\theta} s \theta s \phi c \phi-\dot{\phi} c 2 \phi c \theta & \dot{\theta} c \theta \\
\dot{\theta} s c \theta-\dot{\phi} c 2 \phi c \theta & \dot{\phi} s 2 \phi & 0 \\
\dot{\theta} c \theta & 0
\end{array}\right] \\
& \left\{\begin{array}{l}
C_{1,1}=C_{1,2}=C_{1,3}=0 \\
C_{2,1}=I_{x x}\left(\dot{\psi} c_{\phi}^{2} s 2 \theta+\dot{\theta} s \phi c \phi s \theta-\dot{\phi} c \theta\right) \\
C_{2,2}=I_{x x} \dot{\psi} s \phi c \phi s \theta \\
C_{2,3}=-I_{x x} \dot{\psi} c \theta \\
C_{3,1}=-I_{x x}\left(\dot{\psi} c_{\theta}^{2} s 2 \phi+\dot{\theta} c \theta c 2 \phi\right) \\
C_{3,2}=-I_{x x}(\dot{\psi} c \theta c 2 \phi-\dot{\theta} s 2 \phi) \\
C_{3,3}=0
\end{array}\right.
\end{aligned}
$$

where $m$ denotes the mass of the rotorcraft and $I_{X X}=I_{Y Y}=m l^{2}, I_{Z Z}=2 m l^{2}$.

Where (see Figure 1) [20, 21]:

$$
\begin{aligned}
& u_{1}=F_{1}+F_{2}+F_{3}+F_{4} \\
& u_{4}=d\left(F_{1}-F_{2}+F_{3}-F_{4}\right) \\
& u_{3}=\left(F_{2}-F_{4}\right) l \\
& u_{2}=\left(F_{3}-F_{1}\right) l
\end{aligned}
$$

Table 1 The parameters of the quadrotor rotorcraft [22]

\begin{tabular}{|c|c|c|}
\hline Definition & Parameter & Value \\
\hline Lever length & $\mathrm{l}$ & $0.232 \mathrm{~m}$ \\
\hline Mass of Quadrotor & $\mathrm{m}$ & $0.52 \mathrm{~kg}$ \\
\hline Drag coefficient & $\mathrm{d}$ & $7.5 \mathrm{e}-7 \mathrm{~N} \mathrm{~ms} 2$ \\
\hline Thrust coefficient & $\mathrm{b}$ & $3.13 \mathrm{e}-5 \mathrm{~N} \mathrm{~s} 2$ \\
\hline Rotor inertia & $\mathrm{Jr}$ & $6 \mathrm{e}-5 \mathrm{~N} \mathrm{kgm} 2$ \\
\hline $\begin{array}{c}\text { Gravitational } \\
\text { acceleration }\end{array}$ & $\mathrm{g}$ & $9.81 \mathrm{~m} / \mathrm{s} 2$ \\
\hline
\end{tabular}

\section{Ftc Strategy}

In this section, adaptive PID controllers were to best approximate the ideal command unknown (14) [24].

$$
u=u^{*}=\frac{(-f(x)+v+\alpha s+\beta \tanh (s / \varepsilon))}{g(x)}
$$

with $\alpha>0, \beta>0$ and $\varepsilon$ a small positive constant.

Where:

$$
s(t)=\left(\frac{d}{d t}+\lambda\right)^{n-1} e(t), \quad \lambda>0
$$

where: $e(t)=y_{d}(t)-y(t)$.

Choose the Lyapunov function:

$$
V=\frac{1}{2} s^{2}
$$

The derivative of (10) along paths (9) is bounded by:

$$
\dot{V} \leq-\alpha s^{2}
$$

The derivative of the filtered error can be written as [24]

$$
\dot{s}=v-f(x)-g(x) u
$$

with: $\quad v=y_{d}{ }^{(n)}+k_{n-1} e^{(n-1)}+\ldots+k_{1} \dot{e}, \quad k_{j}=C_{n-1}^{j-1} \lambda^{n-j}$ where $\lambda$ is positive constant.

The three PID controller gains $k_{p}, k_{i}$ and $k_{d}$ were considered here as adjustable parameters. To do this, an adaptive mechanism would be developed to minimize a quadratic criterion of the error between the ideal unknown command $u^{*}$ and the command $u_{\text {pid }}$ provided, resulting from the PID controller. The ideal control law (14) was then approximated by a PID controller of the form.

$$
u=u_{p i d}=k_{p} e(t)+\mathrm{k}_{i} \int_{0}^{t} e(\tau) d \tau+k_{d} \frac{d e(t)}{d t}
$$

where: $u=\prod^{T}(e) \theta_{l}$.

$$
\prod(e)=\left[\begin{array}{lll}
e(t) & \int_{0}^{t} e(\tau) d \tau & \frac{d e(t)}{d t}
\end{array}\right]^{T}
$$

$\theta_{l}$ is the vector of parameters adjusted in the control, which is defined by: $\theta_{l}=\left[\begin{array}{lll}k_{p} & k_{i} & k_{d}\end{array}\right]$ 
From equation (18)

$$
\dot{s}=-\alpha s-\beta \tanh (s / \varepsilon)+g(x)\left(u-u^{*}\right)
$$

with: $u^{*}=\prod^{T}(e) \theta_{l}^{*}$ is optimal command and $\theta^{*}$ is the optimal parameters.

From equation (21)

$$
g(x) e_{u}=\dot{s}+\alpha s+\beta \tanh (s / \varepsilon)
$$

From (22), the law of adaptation parameters is given by

$$
\dot{\theta}_{l}=v \prod(e)\{\dot{s}+\alpha s+\beta \tanh (s / \varepsilon)\}
$$

\section{Simulation Result}

The proposed LGS-PID control scheme (fig.2) presented in this paper was tested on a model of the full Quadrotor helicopter model in presence of the actuators faults.

It is assumed that a loss of control effectiveness of $40 \%$ by echelon the faults were taking place in the command $u_{1}, u_{2}, u_{3}$ and $u_{4}$ at time instant $t=35 \mathrm{~s}$ and ends on time $t=55 \mathrm{~s}$.

The synthesis parameters are selected as Table 2 .

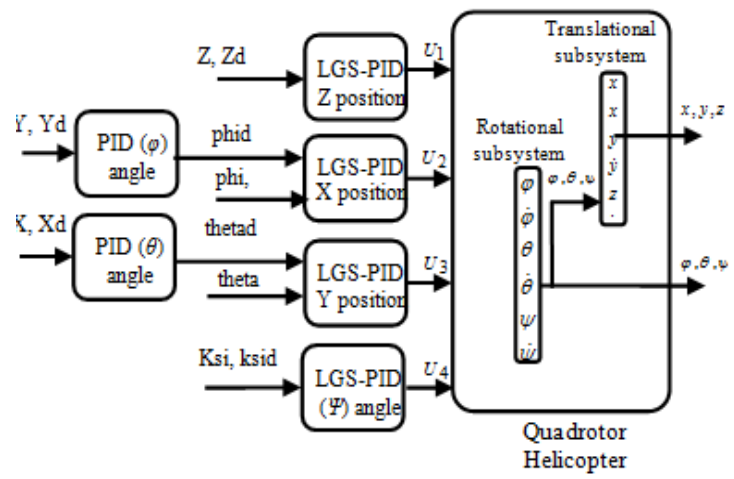

Figure 2 Synoptic scheme of the proposed control strategy

\begin{tabular}{|c|c|c|}
\hline Definition & Parameter & Value \\
\hline $\begin{array}{l}\mathrm{PID}(\varphi) \\
\text { angle }\end{array}$ & $K_{p}, K_{i}, K_{d}$ & $\begin{array}{c}K_{p}=4.5, K_{i}=1.5, K_{d} \\
=1.5\end{array}$ \\
\hline $\begin{array}{c}\text { PID }(\theta) \\
\text { angle }\end{array}$ & $K_{p}, K_{i}, K_{d}$ & $\begin{array}{c}K_{p}=3.5, K_{i}=0.5, K_{d} \\
=2\end{array}$ \\
\hline \multirow{3}{*}{$\begin{array}{l}\text { LGS-PID } \\
Z \text { position }\end{array}$} & $K_{p}$ & $\begin{aligned} \alpha=20, \varepsilon & =0.001, \beta=10, \\
v & =3, \lambda=5\end{aligned}$ \\
\hline & $K_{i}$ & $\begin{array}{c}\alpha=10, \varepsilon=0.001, \beta=20, \\
v=4, \lambda=20\end{array}$ \\
\hline & $K_{d}$ & $\begin{array}{c}\alpha=18, \varepsilon=0.001, \beta=2, \\
v=0.5, \lambda=1\end{array}$ \\
\hline LGS-PID & $K_{p}$ & $\alpha=22, \varepsilon=0.001, \beta=12$, \\
\hline
\end{tabular}

Table 2 Synthesis parameters of the proposed controller

\begin{tabular}{|c|c|c|}
\hline$X$ position & & $v=1.27, \lambda=2.1$ \\
\cline { 2 - 3 } & $K_{i}$ & $\begin{array}{c}\alpha=20, \varepsilon=0.001, \beta=2, \\
v=1.4, \lambda=2\end{array}$ \\
\cline { 2 - 3 } & $K_{d}$ & $\begin{array}{c}\alpha=8, \varepsilon=0.001, \beta=1.2, \\
v=5, \lambda=3\end{array}$ \\
\hline \multirow{4}{*}{$\begin{array}{c}\text { LGS-PID } \\
Y \text { position }\end{array}$} & $K_{p}$ & $\begin{array}{c}\alpha=12, \varepsilon=0.001, \beta=6, \\
v=3.7, \lambda=2\end{array}$ \\
\cline { 2 - 3 } & $K_{i}$ & $\begin{array}{c}\alpha=13, \varepsilon=0.001, \beta=3.7, \\
v=5.4, \lambda=2.5\end{array}$ \\
\cline { 2 - 3 } & $K_{d}$ & $\begin{array}{c}\alpha=9, \varepsilon=0.001, \beta=2, \\
v=5.2, \lambda=2\end{array}$ \\
\hline & $K_{p}$ & $\begin{array}{r}\alpha=7, \varepsilon=0.001, \beta=4, \\
v=7, \lambda=1.7\end{array}$ \\
\cline { 2 - 3 } $\begin{array}{c}\text { LGS-PID } \\
\Psi \text { angle }\end{array}$ & $K_{i}$ & $\begin{array}{r}\alpha=8, \varepsilon=0.001, \beta=3, \\
v=5, \lambda=2\end{array}$ \\
\cline { 2 - 3 } & $K_{d}$ & $\begin{array}{r}\alpha=11.4, \varepsilon=0.001, \beta=2, \\
v=2, \lambda=1\end{array}$ \\
\cline { 2 - 3 } & &
\end{tabular}
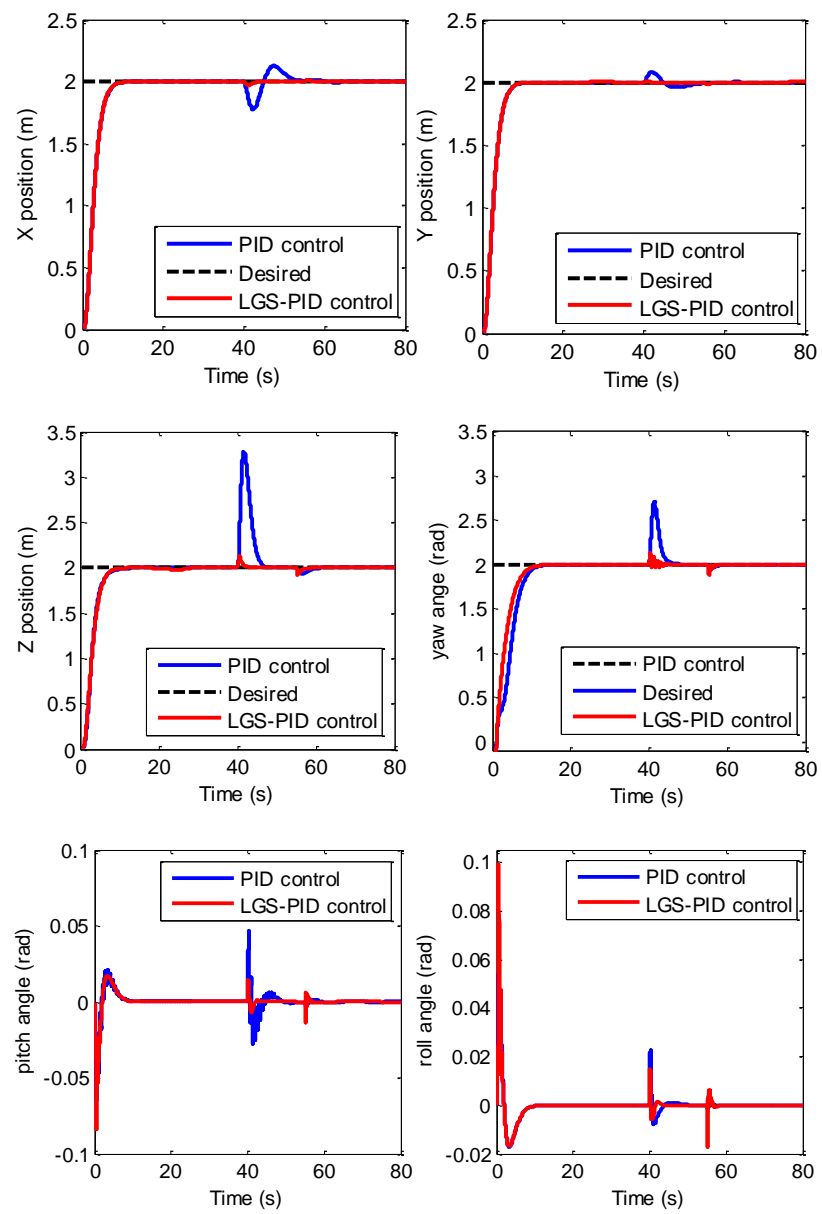

Figure 3 Comparison between PID control and LGS-PID control 

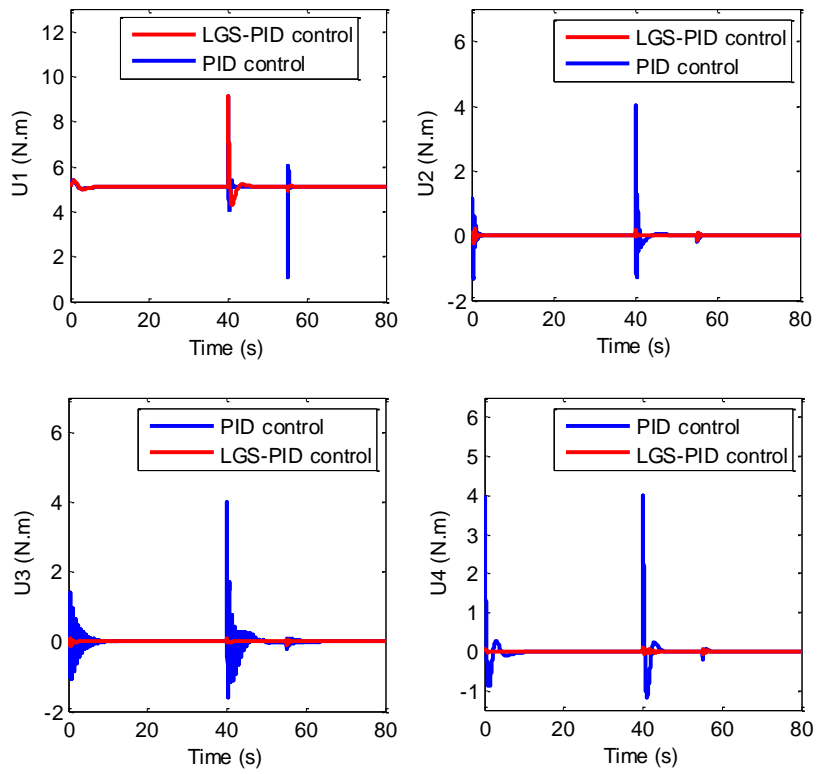

Figure 4 Commands $u_{1}, u_{2}, u_{3}$ and $u_{4}$ of PID control and LGS-PID control
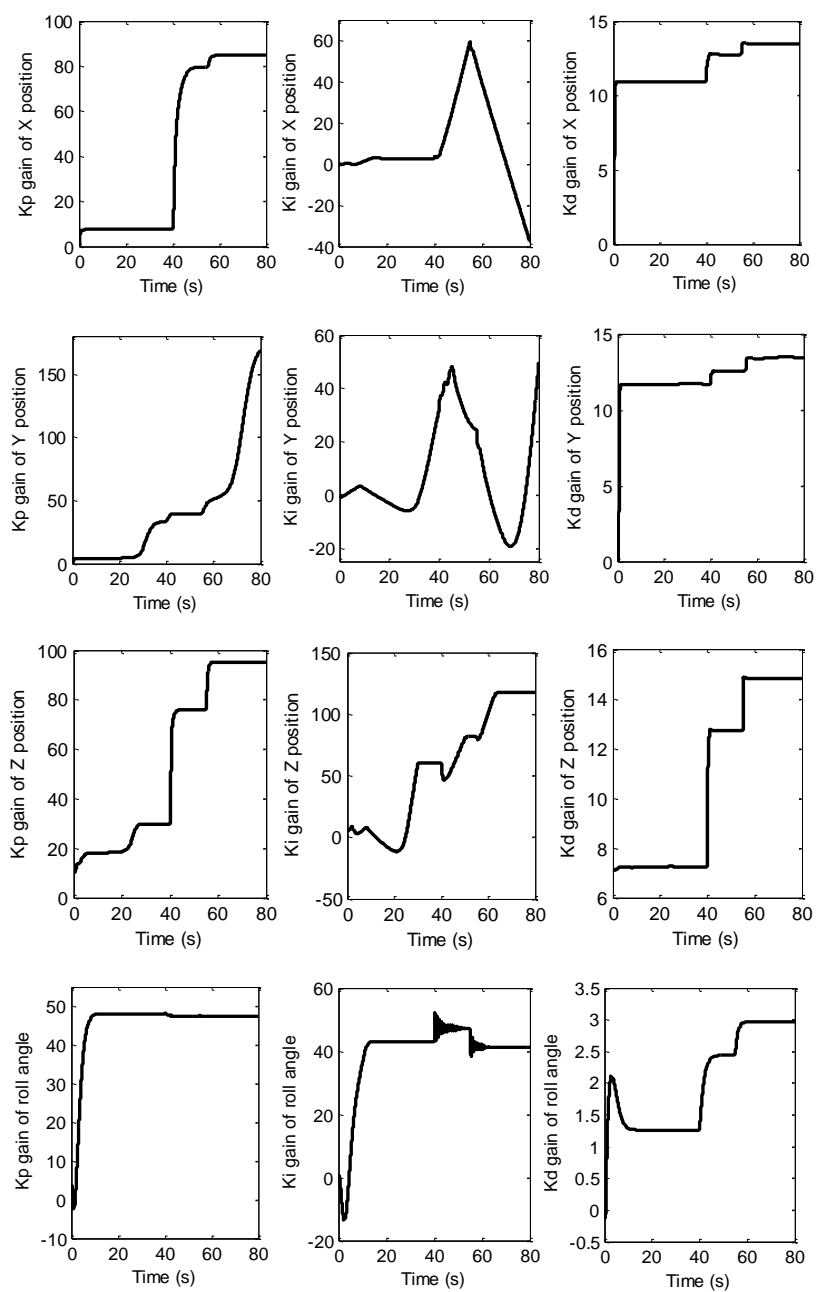

Figure 5 Gains $K_{p}, K_{i}$ and $K_{d}$ of LGS-PID control

The time evolutions of the LGS-PID gains are illustrated in Figure 5. Unlike those of the PID control, the LGS-PID gains were time-varying to adapt to uncertainties, disturbances as can been clearly in figure 6. It can be seen in Figure 3, 4 and 5 that after the fault occurs, $K_{p}$ decreased to avoid system pass due to increase in tracking error. The derivative gain $K_{d}$ remained fixed with a high value to make a fast response to sudden changes in tracking error. When the system stopped descending (losing altitude) $K_{d}$ decreased to let the system recover faster and go back to its desired position. After the fault, integrator gain $K_{i}$ also increased to help the recovery process.

\section{Conclusion}

In this paper, we presented the Fault-Tolerant Lyapunov-Gain-Scheduled PID Control of the full Quadrotor helicopter in the presence of the fault. Firstly, we started by the development of the dynamic model of the Quadrotor taking into account the different physics phenomena, after we are interested in proposing the FTC controller based on Lyapunov method. Simulation results also validated that the presented FTC had a satisfactory tracking performance and was robust to the external disturbances.

\section{References}

[1] M. Witczak, V. Puig, and S. Montes de Oca, "A fault-tolerant control scheme for non-linear discrete- time systems: Application to the twin-rotor system”, In: Conf. Rec. 2010 IEEE Int. Conf. Control and Fault-Tolerant Systems (SysTol), pp.861-866, 2010.

[2] Ł.Dziekan, M.vWitczak and J. Korbicz, "Active fault-tolerant control design for Takagi-Sugeno fuzzy systems", Bulletin of the polish academy of sciences technical sciences, Vol.59, No.1, 2011.

[3] J. Chen, R. Patton and Z. Chen, "An LMI approach to fault tolerant control of uncertain systems", In: Conf. Rec. 1998 IEEE Int. Conf. Decision and Control, pp.175-180, 1998.

[4] Z. Qu, C. Ihlefeld, J. Yufang and A. Saengdeejing, "Robust fault-tolerant self-recovering control of non-linear uncertain systems", Automatica. Vol.39, pp.1763-1771, 2003.

[5] J. G. Ziegler and N. B. Nichols, "Optimum settings for automatic controllers", ASME Trans. Vol.64, pp. 759-768, 1942.

[6] T. C. T. Ng, F. H. F. Leung and P. K. S. Tam, "A simple gain scheduled PID controller with stability consideration based on a grid-point concept", In: Conf. Rec. 1997 IEEE Int. Conf. Industrial Elec- 
tronics, pp.1090-1094, 1997.

[7] F. Karray, W. Gueaieb and S. Al-Sharhan, "The hierarchical expert tuning of PID controllers using tools of soft computing" IEEE Trans. Systems Man, and Cybernetics-Part B: Cybernetics, Vol.32, No.1, pp.77-90, 2002.

[8] Z. Zhao, M. Tomizuka and S. Isaka, "Fuzzy gain scheduling of PID controllers", IEEE Trans. on Systems Man and Cybernetics, Vol.23, No.5, pp. 1392-1398, 1993.

[9] K. Yu and J. Hsu, "Fuzzy gain scheduling PID control design based on particle swarm optimization method" In: Second International Conference on Innovative Computing, Information and Control. Kumamoto, 2007.

[10] Zulfatman and M. F. Rahmat, "Application of selftuning fuzzy PID controller on industrial hydraulic actuator using system identification approach", Int. J. on Smart Sensing and Intelligent Systems, Vol.2, pp.246-261, 2009.

[11] Y. Guo and T. Yang, "A new type of computational verb gain-scheduling PID controller", In: International Conference on Counterfeiting Security and Identification in Communication, Chengdu, pp. 235-238, 2010.

[12] L. Yao and C. Lin, "Design of gain scheduled fuzzy PID controller", World Academy of Science, Engineering and Technology, Vol.1, pp.152-156, 2005.

[13] I. M. M. El Emary, W. Emar and M. J. Aqel, "The adaptive fuzzy designed PID controller using wavelet network", Journal of Computer Science and Information System, Vol.6, No.2, pp.141-163, 2009.

[14] J. Jih-Gau and T. Kai-Ti, "Design and realization of a hybrid intelligent controller for a twin rotor mimo system", Journal of Marine Science and Technology, Vol.21, No.3, pp.333-341, 2013.

[15] B. Hu, G. K. I. Mann and R. G. Gosine, "A systematic study of fuzzy PID controllers-function based evaluation approach", IEEE Trans. on Fuzzy Systems, Vol.9, No.5, pp.699-712, 2001.

[16] J. T. Spooner and K. M. Passino, "Stable adaptive control using fuzzy systems and neural networks", IEEE Trans. on Fuzzy Syst., Vol.4, No.3, pp. 339-359, 1996.

[17] W. D. Chang, R. C. Hwang, and J. G. Hsieh, "A self-tuning PID control for a class of nonlinear systems based on the Lyapunov approach", Journal of Process Contr., Vol.12, No.2, pp.233-242, 2002.

[18] M. Feki, "An adaptive feedback control of linearizable chaotic systems," Chaos, Solitons and Fractals, Vol.15, No.5, pp.883-890, 2003.
[19] S. Bouabdallah and R. Siegwart, "Backstepping and Sliding-mode Techniques Applied to an Indoor Micro Quadrotor", In: Conf. Rec. 2005 IEEE Int. Conf. Robotics and Automation, Barcelona, Spain, pp.2247-2252, 2005.

[20] R. Xu and U. Ozguner, "Sliding mode control of a Quadrotor helicopter", In: Conf. Rec. 2006 IEEE Int. Conf. Decision \& Control, San Diego, USA, pp. 4957-4962, 2006.

[21] M. Önder Efe, "Robust low altitude behavior control of a Quadrotor rotorcraft through sliding modes", In: Conf. Rec. 2007 Mediterranean. Control and Automation, Athens, Greece, pp.23-35, 2007.

[22] P. Castillo, R. Lozano and A. E. Dzul, "Modeling and Control of Mini-Flying Machines", SpringerVerlag London Limited 2005.

[23] A. Boubakir, S. Labiod and T. M. Guerra, "Commande PID Adaptative des Systèmes non Linéaires Affines en la Commande", CGE'06, EMP, ALGER, April, 2009 